\title{
Isolated anti-Ku antibody in scleroderma-myositis overlap syndrome: the histo-pathological patern
}

\author{
Bernard Azanmene ${ }^{1 *}$, Valerie Badot ${ }^{1}$, Severine Verlinden ${ }^{1}$, Maria Josee Fernandez-Lopez ${ }^{1}$, Hazim Kadhim², \\ Francis Corazza ${ }^{3}$, Wolfram Fink ${ }^{4}$, Anne Peretz ${ }^{1}$, Jacques Bentin ${ }^{1}$ \\ From 7th European Workshop on Immune-Mediated Inflammatory Diseases \\ Noordwijk aan Zee, the Netherlands. 28-30 November 2012
}

An 18-years old African girl, suffered for almost 4 years of polyarthralgia, joint contractures and a hardcover appearance of the skin suggestive of scleroderma. The clinical picture and a high level of CPK suggested Polymyositis (PM).

Search for anti nuclear Antibody (ANA) showed speckled pattern at $1 / 2500$ titre, and subscreening revealed isolated anti-Ku antibody. This was more often reported in association with PM - SSc overlap syndrome.

Skin biopsy favored linear scleroderma.

Interestingly, muscle biopsy showed features typical of dermatomyositis (DM).

No endocrine disorder or underlying mitotic process was registered.

Corticosteroid therapy was initiated followed by maintenance therapy with methotrexate; the outcome was favorable.

This case illustrates the interest of anti-Ku screening in the diagnostic work-up. Our observation thus emphasizes the possible occurrence of isolated anti-Ku antibody expression in an overlap syndrome comprising SSc-DM association. Such association/linkage (implicating DM rather than PM) is, to our knowledge, very rarely well reported. Moreover, the therapeutic response seems favorable in such condition.

We found one biopsy-confirmed observation of SScInflammatory myositis (IM) overlap syndrome in an anti$\mathrm{Ku}$ positive patient $[1,2]$. This later case was initially reported as SSc-PM overlap syndrome. Interestingly, follow-up showed evolution towards typical features of DM, and hence an overlap syndrome of SSc-DM association.

Thus, our observation highlights the necessity/interest of biopsy to ascertain the precise nature of myositis in an overlap syndrome associating SSc and Myositis Clinico-pathological criteria in inflammatory myositis have been reviewed by Chérin $P$ et al., [3].

Anti-ku Ab is knowingly reported in a context of myositis. Thus our observation and review of the literature suggest that in the presence of isolated anti-Ku $\mathrm{Ab}$ in a SSc-Myositis overlap context, muscle biopsy (that should be done) would tend to show DM (rather than PM) in association with the SSc. Such a finding may change the prognosis and the treatment approach of this syndrome. This can only be demonstrated after elaborate muscle biopsy in overlap syndromes, with or without the presence of anti-Ku antibodies.

\section{Author details}

'Dept. of Rheumatology, Brugmann University Hospital (U.L.B.), Brussels, Belgium. ${ }^{2}$ Dept. of Pathology, Brugmann University Hospital (U.L.B.), Brussels, Belgium. ${ }^{3}$ Dept. of Clinical Biology, Brugmann University Hospital (U.L.B.), Brussels, Belgium. ${ }^{4}$ Dept. of Dermatology, Brugmann University Hospital (U.L. B.), Brussels, Belgium.

Published: 28 November 2012

\section{References}

1. Yamanishi Y, Maeda H, Katayama S, Ishioka S, Yamakido M: Sclerodermapolymyositis overlap syndrome associated with anti-Ku antibody and rimmed vacuole formation. J Rheumatol 1996, 23:1991-1994.

2. Kamei N, Yamane K, Yamashita Y, et al: Anti-Ku antibody-positive scleroderma-dermatomyositis overlap syndrome developing Graves' disease and immune thrombocytopenic purpura. Intern Med 2002, 41:1199-1203.

3. Chérin $P$, Marie I: Les nouveaux critères diagnostiques et d'évaluation des polymyosites et dermatomyosites. Rev Med Interne 2005, 26(5):361-7, Epub 2005 Mar 17.

doi:10.1186/1479-5876-10-S3-P57

Cite this article as: Azanmene et al:: Isolated anti-Ku antibody in scleroderma-myositis overlap syndrome: the histo-pathological patern. Journal of Translational Medicine 2012 10(Suppl 3):P57. 